Document downloaded from:

http://hdl.handle.net/10251/146360

This paper must be cited as:

Prodaniuc, C.; Stojanovic, N.; Karinou, F.; Goeger, G.; Qiang, Z.; Llorente, R. (2015). 56 Gb/s, PAM-4 Transmission Over 25 km, Using IQ Modulator and Unequally Spaced Levels. Optical Society (OSA). 1-2. https://doi.org/10.1364/CLEO_SI.2015.STu4F.6

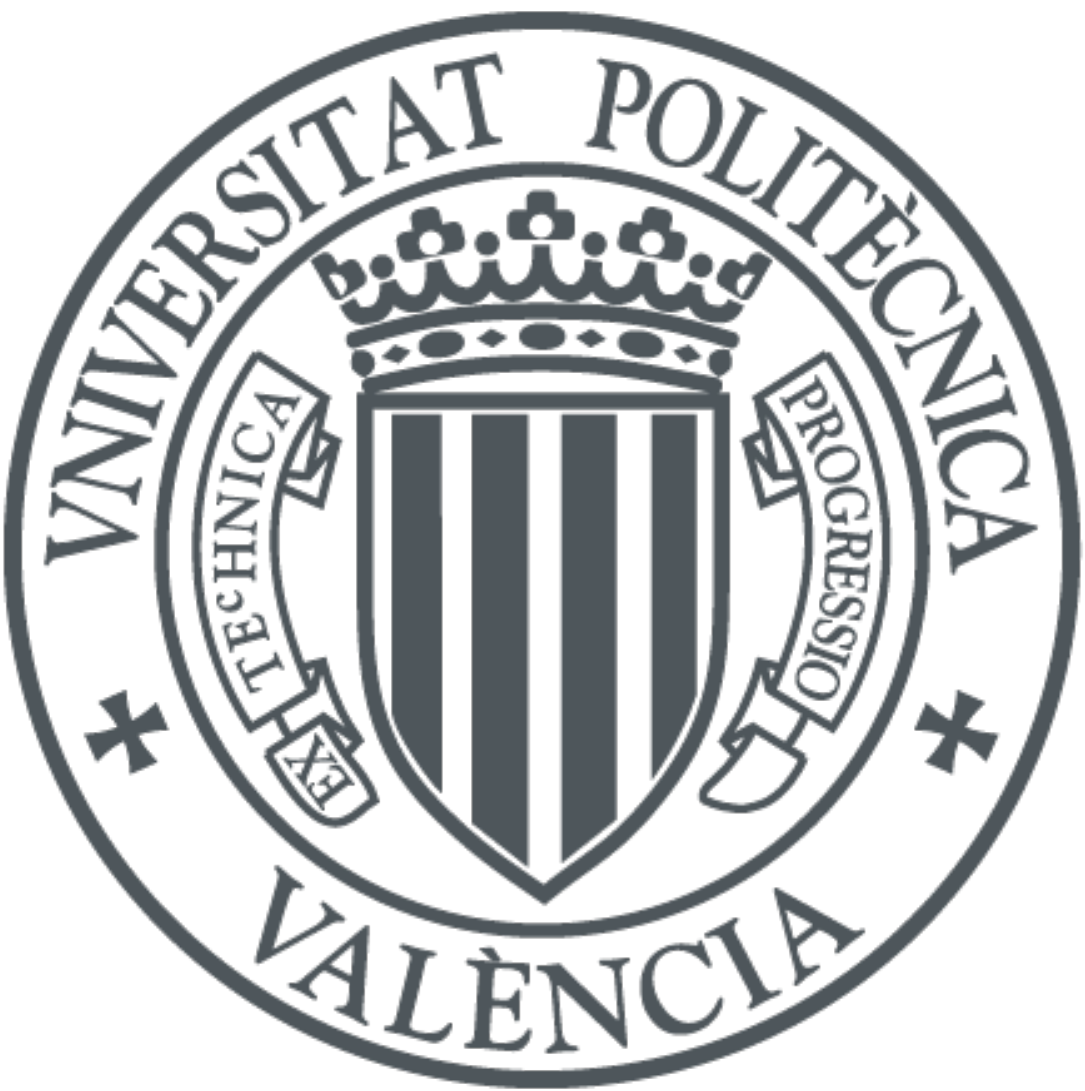

The final publication is available at

https://doi.org/10.1364/CLEO_SI.2015.STu4F.6

Copyright Optical Society (OSA)

Additional Information 


\title{
56 Gb/s, PAM-4 Transmission Over 25 km, Using IQ Modulator and Unequally Spaced Levels
}

\author{
Cristian Prodaniuc $^{1), 2)}$, Nebojsa Stojanovic ${ }^{1}{ }^{\text {, }}$, Fotini Karinou ${ }^{1}$, Gernot Goeger ${ }^{1)}$, Zhang Qiang ${ }^{1)}$, \\ Roberto Llorente ${ }^{2)}$ \\ 1) Huawei Technologies Düsseldorf GmbH, München, Germany \\ 2) Nanophotonics Technology Centre, Universidad Politécnica de Valencia, Spain \\ cristian.prodaniuc@huawei.com,nebojsa.stojanovic@huawei.com,rllorent@dcom.upv.es
}

\begin{abstract}
We propose a PAM-4 modulation scheme with unequally spaced levels in order to mitigate chirp effects caused by IQ modulators. Experimental results of $56 \mathrm{~Gb} / \mathrm{s}$ transmission over $25 \mathrm{~km}$ link demonstrate the robustness of this modulation concept. OCIS codes: (060.4080) Modulation, (060.4510) Optical communications
\end{abstract}

\section{Introduction}

For short range and metro-access optical transmissions, low cost solutions are an attractive alternative to coherent systems. In recent years, Intensity modulation and direct detection (IM-DD) systems have become very attractive due to their simplicity, power dissipation and cost [1]. Most of these systems employ non-return to zero on-off shift keying (NRZ-OOK) modulation, which is simple to implement and has very good optical to noise ratio (OSNR) tolerance. However, with the ever increasing demand for higher line rates, multilevel modulation becomes more attractive [2, 3]. Higher level IM formats such as pulse amplitude modulation 4 (PAM-4) provide the same bit rate as a NRZ-OOK format at lower baud rates, thus reducing the negative dispersive effects of the fiber and facilitating optical transmissions at bit rates of $56 \mathrm{~Gb} / \mathrm{s}$ or higher $[3,4]$.

In this paper we investigate the performance of PAM-4 at $56 \mathrm{~Gb} / \mathrm{s}$ data rate when using an IQ Mach-Zehnder modulator (MZM) to generate the optical signal. We believe that IQ modulators, while not the cheapest alternative, will become the main option in future flexible optical systems. We propose a PAM-4 scheme with unequally spaced levels which removes the effects of modulator chirp, thus enabling transmission distances of up to $25 \mathrm{~km}$.

\section{Mitigation of chirp effects}

First, the generation of equally spaced levels PAM-4 using an IQ modulator is presented. Two pseudo random bit sequences (PRBS) are generated. The power level corresponding to bit ' 1 ' $\mathrm{c}$ ' the first PRBS is twice that of the second PRBS, while the power level corresponding to bit ' 0 ' should be zero for both PRBS. The first PRBS enters the I branch of the IQ modulator and the second one is connected to the Q branch, as can be seen in Fig. 1(a):

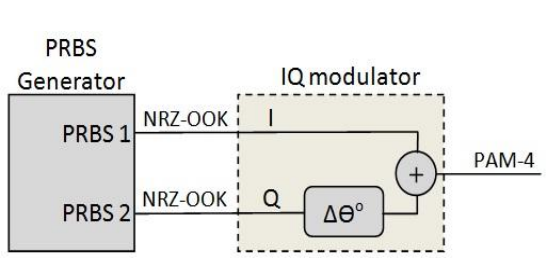

(a)

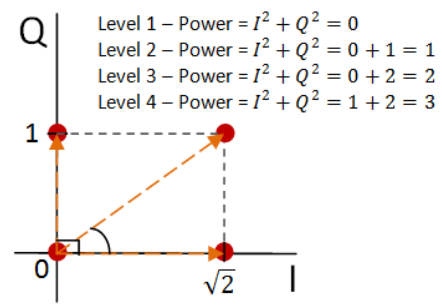

(b)

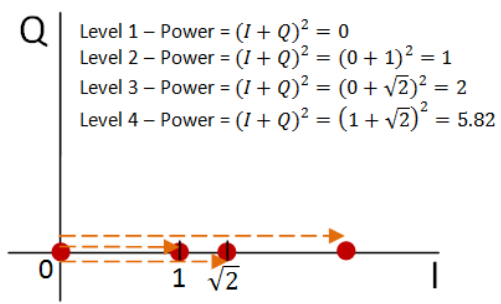

(c)

Fig. 1. (a) Diagram of PAM-4 generation, (b) constellation of equally spaced levels PAM-4 with $\pi / 2$ phase bias, (c) constellation of unequally spaced PAM-4 with zero phase bias.

In order to obtain equidistant power levels at the output of the IQ modulator, the phase bias of the Q branch needs to be $\pi / 2$. Fig. 1(b) shows the resulting constellation. It can be seen that the constellation points are not on the same axis, meaning that the signal is modulated in both intensity and phase domains. In an IM-DD system the phase modulation introduces chirp, which causes additional inter-symbol interference (ISI) when the signal is affected by chromatic dispersion (CD). To counter this phenomenon, the phase bias between the two branches of the modulator must be set to zero. By doing this, it becomes impossible to generate an optical PAM-4 signal with equally spaced levels and thus the unequally spaced levels are obtained. In a back-to-back (BTB) scenario this approach will provide worse bit-error rate (BER) than the equidistant levels PAM-4 for the same OSNR requirements. However, when propagating through standard single mode fiber (SSMF), the drop in performance is smaller in the case of unequally spaced levels PAM-4 and after a certain link distance it will provide better BER than its counterpart [5]. 


\section{Experimental Setup and Results}

Fig. 2 shows the experimental setup used for the investigation of the two different PAM-4 implementations:

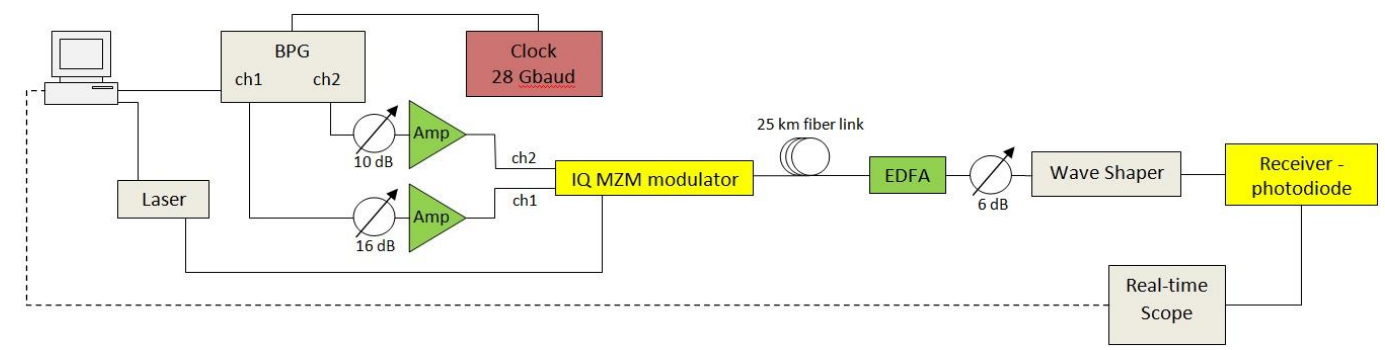

Fig. 2. Block diagram of experimental setup - 28 Gbaud, PAM-4, link up to $25 \mathrm{~km}$.

A bit pattern generator (BPG) is used to generate two NRZ-OOK signals with the baud rate of 28 Gbaud. One channel is attenuated by $6 \mathrm{~dB}$ in respect to the other. After $24 \mathrm{~dB}$ amplification, the two signals will have the appropriate peak-to-peak voltage $(2 \mathrm{~V}$ and $1 \mathrm{~V})$ in order to operate the MZM in the linear region. The resulting constellation of the unequally spaced levels PAM-4 is shown in Fig. 3(c). After passing through the SSMF fiber, the PAM-4 signal is detected by an InGaAs photodiode and sampled by a real-time scope at $50 \mathrm{GSamples} / \mathrm{s}$. The performance of the two PAM-4 modulation schemes evaluated at link lengths of up to $25 \mathrm{~km}$ is presented in Fig. 3(a), where maximum likelihood sequence estimator (MLSE) equalizer has been used:

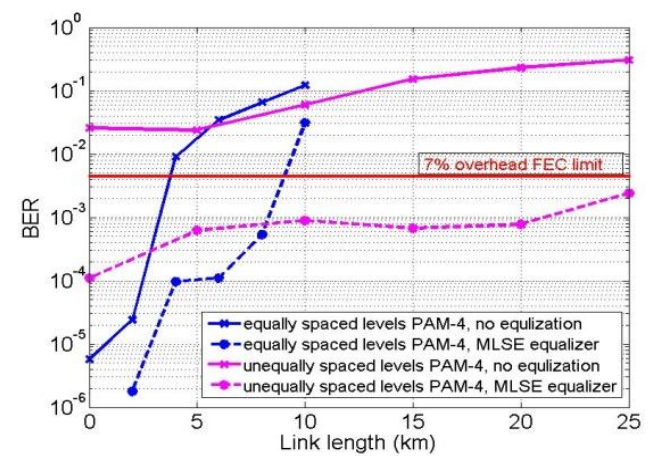

(a)

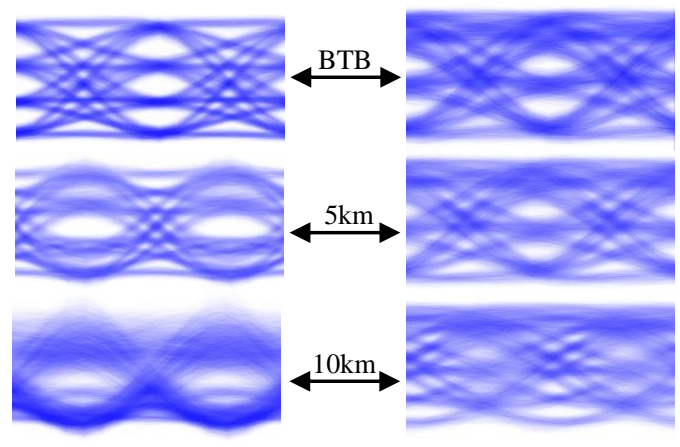

(b)

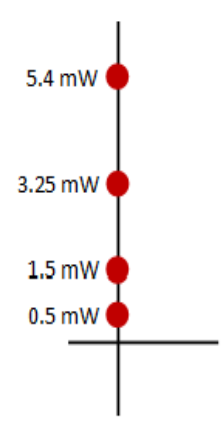

(c)

Fig. 3. (a) Performance comparison between equally spaced levels (blue) and unequally spaced levels (magenta) PAM-4, (b) received eye diagrams for equally spaced levels (left column) and unequally spaced levels (right column) PAM-4 after 0,5 and $10 \mathrm{~km}$, (c) and the unequally spaced levels PAM-4 constellation with corresponding optical power levels (at the receiver the levels will be inversed).

It can be seen that for short transmission distances of up to $8 \mathrm{~km}$ the equidistant levels PAM-4 performs better. However, for links of $10 \mathrm{~km}$ or longer, this scheme cannot achieve BER below the forward error correction (FEC) threshold of 0.004 (7\% Super-FEC). On the other hand, the unequally spaced levels modulation scheme is performing better after $8 \mathrm{~km}$ and can extend the transmission length to $25 \mathrm{~km}$. The eye diagrams in Fig. 3b also show the difference between received signals affected by chirp (left side) and signals that are chirp free (right side).

\section{Summary}

We have indicated the chirp disruptive effects introduced by an IQ modulator used for generating PAM-4 after propagating through SSMF. A novel method based on unequally spaced levels that solves the chirp problem is proposed. We experimentally demonstrated the advantages and disadvantages of this solution and shown that transmission lengths of up to $25 \mathrm{~km}$ at $56 \mathrm{~Gb} / \mathrm{s}$ are possible using this novel PAM-4 modulation scheme.

\section{Reference}

[1] G. Agrawal, "Fiber-Optic Communication Systems," 4th Edition, Wiley, 2012.

[2] Jiangwei Man, Wei Chen, Xiaolu Song, and Li Zeng, "A Low-Cost 100GE Optical Transceiver Module for 2km SMF Interconnect with PAM4 Modulation," OFC 2014, paper M2E.7, 2014.

[3] Lau F. Suhr, J.J. Vegas Olmos, B. Mao, X. Xu, G. N. Liu, and I. Tafur Monroy, “112-Gbit/s x 4-Lane Duobinary-4-PAM for 400GBase,” ECOC 2014, paper Tu.4.3.2, 2014

[4] Krzysztof Szczerba, Petter Westbergh, Magnus Karlsson, Peter A. Andrekson, and Anders Larsson, "70 Gbps 4-PAM and 56 Gbps 8-PAM using an $850 \mathrm{~nm}$ VCSEL," ECOC 2014, paper Tu.4.3.4, 2014

[5] Annika Dochhan, Majed Omar Al-Dwairi, and Werner Rosenkranz, "Optimization of Cost Efficient Multilevel-ASK Modulation Formats under the Constraint of Chromatic Dispersion,” OFC 2010, paper OMJ7, 2010. 